\title{
Underwater Computing Systems and Astronomy-Multi-Disciplinary Research Potential and Benefits
}

\author{
Ayodele Periola*, Akintunde Alonge, Kingsley Ogudo \\ University of Johannesburg, Electrical and Electronic Engineering Technology, Johannesburg, 2092, South Africa
}

\begin{tabular}{l} 
A R T I C L E I N F O \\
\hline Article history: \\
Received: 16 November, 2020 \\
Accepted: 27 January, 2021 \\
Online: 16 February, 2021 \\
\hline
\end{tabular}

Keywords:

Astronomy

Underwater Camera Networks

Computing

Technological Development

Performance Improvement

\begin{abstract}
A B S T R A C T
The Ocean plays an important role in hosting investigations in underwater astronomy and enabling the realization of new research prospects. This paper discusses synergistic prospects of the blue economy from the perspective of underwater astronomy and scientific inquiry, technological and economic development. The presented research investigates how the synergy enhances computing applications. The paper presents the overloaded application paradigm that explores the ability of underwater telescope networks to accommodate additional applications. The investigated metrics for computing applications are the accessible computational resources, and power usage effectiveness (PUE) that is investigated in a scenario where onshore computing stations used in underwater astronomy observations are integrated with existing terrestrial data centers. This is necessary as onshore computing stations benefit from free cooling being located near natural maritime resources. The performance evaluation investigates how the proposed synergy and the emerging crowd-sourcing can enhance the observation resolution for underwater astronomy observations. Investigation shows that the synergy enhances accessible computational resources, PUE and observation resolution by an average of $48.8 \%, 1.6 \%$ and $41.3 \%$, respectively.
\end{abstract}

\section{Introduction}

Knowledge discovery and the conduct of research is an important goal that can be realized via the emergence of new paradigms that aim to answer existing and new research questions. An important research aspect in this regard is that of astronomy observations. The role of the ocean in expanding research frontiers is relatively under-explored in comparison to earth's terrestrial environment with respect to astronomy. There are different forms of astronomy such as optical astronomy, X-ray astronomy, gamma astronomy, and radio astronomy.

A new frontier for the conduct of astronomy is the ocean that enables the realization of underwater astronomy. Underwater astronomy focuses on the study of neutrinos and uses underwater telescopes (i.e., scientific underwater vehicles). The conduct of underwater astronomy requires the launch of underwater telescopes (scientific underwater vehicles) into the ocean at varying locations and altitudes. In underwater astronomy, the concerned telescopes are organized into a network that observes different aspects of the underwater environment with the aim of detecting neutrinos.

\footnotetext{
${ }^{*}$ Corresponding Author: Ayodele Periola, periola@hotmail.com
}

In similarity to other aspects of astronomy, underwater astronomy is a scientific endeavour that aims to advance human knowledge. This can create a false notion that underwater astronomy does not enhance the conduct of other technologies and applications. The result of this is a reduced participation in the conduct of underwater astronomy observation. This leads to lack of or reduced collaboration between scientists in astronomy and other disciplines. A significant effect of this reduced or lack of collaboration is an increased paucity of funds required to develop and deploy underwater astronomy systems. The paucity of funds reduces the chances of underwater astronomy systems in realizing its full potential in contributing to knowledge and also enhancing other application areas. It is important to address this challenge as a conduct of astronomy has enabled technological breakthroughs resulting in useful applications in other domains [1-6]. Nevertheless, it is important to note that the breakthroughs in [16] do not consider the new domain of underwater astronomy [7-9].

The discussion in [7-9] presents an outline of the development of the science of underwater astronomy. In addition, the discussion in [9] identifies other sources that were previously thought to be neutrino sources in the universe. Some of these sources are X-ray binaries and gamma ray bursts. In addition, Spiering recognized that the use of underwater neutrino detectors has been considered 
suitable since 1960. However, the consideration of an approach in which underwater astronomy concerns forms a synergy with other applications requires further research consideration.

The focus of the research presented in this paper is to demonstrate the usefulness and value potential of underwater astronomy. The non-consideration of underwater astronomy in [16] alongside the need to demonstrate the usefulness of underwater astronomy to other application is addressed in this paper.

Contribution: The discussion in the presented research focuses on the conduct of underwater astronomy. The research recognizes that it is challenging to provide a societal basis to justify the conduct of scientific investigations via underwater astronomy observations. The provision of a societal basis is important to convince other applications and disciplines to donate resources in designing and deploying systems for conducting underwater astronomy observations. The realization of this goal requires the identification of other applications that benefit from the conduct of underwater astronomy observations. This goal is realized by identifying applications that can potentially benefit from the launch of systems intended for use in underwater astronomy observations. Two applications i.e., underwater surveillance, and computing systems have been identified. The consideration of the applications of underwater surveillance and computing alongside the concerns in underwater astronomy shares the system realization costs amongst these applications. This reduces the cost associated with the realization of underwater astronomy systems. In addition, the conduct of underwater astronomy while accommodating the concerns of underwater surveillance and computing enhances these applications by improving their performance. This paper also explores how the conduct of underwater astronomy observations provides a platform enabling the conduct of scientific enquiry in reducing pseudo-science towards verifying the existence of aquatic humanoids. In addition, the paper presents a case from the public health perspective to justify the need to continue the conduct of investigation in this direction. Furthermore, the consideration of the applications in underwater surveillance, and computing increases the potential of underwater astronomy in other areas leading to an overloaded application context.

The contribution of this paper is the presentation of the overloaded application paradigm. In the proposed overloaded application paradigm, the ability of underwater astronomy observations to provide support to new research motives and computing applications is examined. The contributions of this paper are:

1) Firstly, the paper proposes the probe of the ocean with the aim of enhancing knowledge discovery and reducing the propagation of pseudo - science. This is important in investigating the existence of aquatic humanoids. The proposed investigation aims to answer questions from two perspectives. The first perspective is that of providing scientific and technical answers to a long-standing concern. The second perspective aims to provide a platform to further investigate how aquatic humanoids have developed immune response to marine viral outbreaks. This paper provides and discusses the rationale and presents a design of the system for conducting the proposed scientific investigation.
2) Secondly, the paper recognizes that the deployment of underwater telescopes increases the coverage of the ocean thereby enabling the realization of underwater surveillance applications. The conduct of underwater astronomy also necessitates the design of novel computing networks for data processing. The discussion in this paper proposes a novel underwater surveillance network that re-uses the infrastructure and network deployed to realize underwater astronomy applications. In addition, the paper proposes a novel computing network architecture enabling the integration of onshore computing platforms with existing terrestrial cloud computing platforms. The paper also recognizes the potential of underwater astronomy to spur technological development in other areas.

In addition, the paper formulates and investigates benefits arising from the synergy in the proposed paradigm. The performance benefits are analysed from the perspectives of computing applications and astronomy investigations. The formulated and investigated metrics from the computing perspective are the accessible computing resources and the PUE. The observation resolution metric has received consideration from the astronomy investigation perspective.

\section{Background}

The value potential of conducting astronomy observations with a focus on Africa receives consideration in [10]. In their discussion, astronomy is considered from a broad perspective i.e. it comprises the use of terrestrial and space-based telescopes. Technological advances in astronomy are recognized to enhance education (for students and teachers), healthcare (eye care), and fostering skills development and; ensure equal gender participation in science, technology, engineering and mathematics.

The discussion in [10] recognizes that additional work is needed to increase astronomy's relevance in Africa. This can be achieved by directing the effort of researchers in astronomy system design to other potential impact areas. The conduct of collaborative research is required to maximize the developmental profits obtainable from astronomy. However, the potential of driving multi-disciplinary research via an examination of multi-domain problems are not done in [10]. This can be addressed in additional research.

The development motive underlying the participation of African nations in Astronomy is discussed in [11]. The Ethiopian initiative uses space science and technology to address challenges being faced in accessing water and enhancing agricultural productivity. The drive by Kenya and Zambia to engage in radio astronomy has motivated the need to develop capacity in converting satellite earth stations to terrestrial radio telescopes. The Sudanese institute of Space Research and Aerospace also aims to establish an astronomy exploration centre and terrestrial telescope. The drive to conduct astronomy research in this case motivates aerial surveillance systems development and; advances small satellite application in education. The drive to conduct astronomy also drives development in remote sensing, satellite meteorology, information and communication technology; and security and defence. 
Astronomy has prospects to enable technological development and advance research in information and communication technology, and security and defence. The discussion in [11] is themed towards developing terrestrial and space-based astronomy assets. It also recognizes the potential of astronomy research to enhance the future of life in Africa. However, this does not consider new areas of astronomy such as aerial astronomy [12] and underwater astronomy observations. The knowledge values and development potential of these aspects should also be examined.

The African Union in [13] discusses the developmental role of space science and astronomy in technological development. The conduct of astronomy has enabled the development of technologies such as the geographical positioning systems, space geodesy, and key roles in analyzing ocean and ice level measurements and continental drifts analysis. The conduct of astronomy observations enables development of conceptual and practical skills that can be transferred to other areas like meteorology, and; information and communication technology. These conceptual skills are useful in other domains such as technology research thereby enabling astronomy to have a significant knowledge value. However, exploring this aspect requires further research.

It is recognized in [14] that most astronomy observatories are located in terrestrial and space-based locations. This view is shared with [11] and [13]. The perspective does not consider underwater astronomy. The conduct of terrestrial astronomy and space astronomy is recognized to drive the development of supercomputing technology and big data analysis methods. Though [12], recognizes the importance and role that terrestrial and space-based astronomy plays in driving technological development; its role in role in designing future computing platforms architecture for distributed terrestrial and space-based astronomy requires further consideration.

The development potential of the square kilometer array is considered in [15] where it is recognized that the square kilometer array enables advances in big data processing algorithms and supercomputing. These developments enhance human capital development and positions South Africa as an emerging knowledge economy. It also enables the emergence of private sector spin-offs that participate in the high-tech global value chain. A similar perspective can be found in [16].

The growing interest in underwater astronomy as seen in [17] positions it to address puzzles in physics such as the origin of the cosmic rays. Developmental efforts are required to realize the design of KM3NeT array [16]. However, lessons that can be learnt from the development of digital optical modules and associated software are not deemed to be useful in addressing underwater research and development concerns.

The study in [18] describes procedures required to determine the suitability of an underwater site for hosting an underwater telescope. A desired site should have suitable optical properties, low background noise and bioluminescence. The optical property of water is influenced by its chemical properties. However, the potential of using the optical properties in other applications has not been examined.
This section shows that the desire to conduct astronomy observations drives technological development. This technology advancing role has been motivated mainly by space-based and terrestrial-based astronomy observations. This has not considered underwater astronomy as recognized and addressed in this paper.

\section{Underwater Astronomy - Support for Scientific Inquiry}

This section explores how ocean exploration and underwater astronomy influences the conduct of investigations in seeking answers to scientific inquiry. It has two aspects. The first and second discuss the motivation for scientific inquiry and the associated system design respectively.

\subsection{Rationale for Scientific Investigation}

The ocean is a vast resource whose exploration is a key goal of scientific investigation. Being explored to a lesser extent in comparison to earth's terrestrial plane, the ocean serves as a platform enabling the conduct of investigations for the purpose of verifying different claims. An important scientific inquiry being considered is verifying the existence of aquatic humanoids biological species. This is deemed necessary to reduce the propagation of pseudo-science [19-21] in the public domain. In addition, the successful detection (thereby proving their existence) or non-existence is deemed important to enhance knowledge discovery in the information age. The discussion in this section also discusses the implications on future global health.

The launch of underwater telescopes enables increased ocean exploration. This provides access to new ocean regions and opportunities to probe for answers to scientific questions such as verifying the actual existence of aquatic humanoids. The research in [20-21] shows that there is the need to conduct additional research to verify this claim. This can potentially provide answers to questions on public health as the ocean is recognized to host a significant number of marine viruses [22-24]. A potential research step is to investigate how aquatic humanoids (if detected) have developed resilience to marine viruses and marine viral outbreaks.

A successful detection of aquatic humanoids is an advantageous step in studying their adapted and developed immune response to viruses. This is helpful in understanding how to develop new systems and solutions to manage viral outbreaks, epidemics and pandemics. A study in this regard is potentially useful in developing solutions to enhance human viral immunity in the future. This is important for future global public health and human welfare.

\subsection{Scientific Investigation-System for Executing Scientific Search}

The execution of a search to scientifically provide an answer on the existence of aquatic humanoids requires a probe of the ocean. This probe can be realized via the launch of scientific payload integrated in a system designed for this purpose. However, this approach incurs a high expense making it prohibitive for developing nations and other capital constrained contexts where underwater telescopes have already been deployed. The approach being proposed in this paper is to re-use the existing framework provided by already deployed underwater telescopes to find answers to the scientific inquiry under investigation. This is being considered as it enables the re-use of hardware and computing 
infrastructure initially deployed for the purposes of underwater astronomy.

Underwater telescopes comprise photomultipliers that are embedded in digital optical modules [25]. These modules are deployed in a string configuration with a pre-defined separating distance. The strings have $15 \mathrm{~m}$ separating distance at depths lying between $745 \mathrm{~m}$ to $1270 \mathrm{~m}$. Additional payload to support other scientific investigations in the underwater environment can be placed in the gap i.e. separating distance lying between digital optical modules. For example, the ANTARES neutrino telescope is at a depth of $2475 \mathrm{~m}$ [26]. The neutrino telescope in [27] is located in the ocean's bathypelagic zone extending between the depths of $1 \mathrm{~km}$ to $4 \mathrm{~km}$. The siting of ANTARES in the bathypelagic zone makes it feasible to conduct ocean related research in this zone. The distance between strings on average in the KM3Net underwater telescope is about $90 \mathrm{~m}$. In addition, the KM3Net underwater telescope has an information and communication network, and supporting infrastructure. The infrastructure is used to transmit acquired results on the scientific search from the ocean.

The payload that executes the search under consideration is hosted in the string gap (separating distance between digital optical modules). It is located in the string gap to prevent disturbance to digital optical module observation. The payload comprises underwater camera (capturing image and video); and underwater acoustic camera. The realization and use of underwater video camera systems is now technologically feasible [28-30]. The underwater video cameras are accompanied with underwater acoustic cameras. The use of underwater acoustic cameras is also feasible because of technology maturation. The underwater acoustic camera detects bio-acoustic signatures from previously undetected biological sources. The underwater video camera [2830] and acoustic underwater camera [30-32] are deployed to scan the underwater environment. The use of the acoustic underwater camera is deemed suitable because biological sources have acoustic bio-signatures [33]. The deployed cameras are immobile and utilize the computing resources aboard the onshore computing station.

A combination of the acoustic bio-signature, picture (alongside video) at a given epoch is sent to the onshore computing facility. Relations between the digital optical modules, underwater (video), underwater acoustic cameras and the onshore computing facility (hosting several servers) is shown in Figure 1. The servers host computing resources used for algorithm execution and data processing. The digital optical modules and cameras are connected to a central module that is linked to the onshore computing facility.

In Figure 1, the cameras are deployed in a network and have varying coverage in various directions within the underwater environment. The deployed cameras and sensors incorporate the benefit of viewing diversity. The limited viewing capabilities of

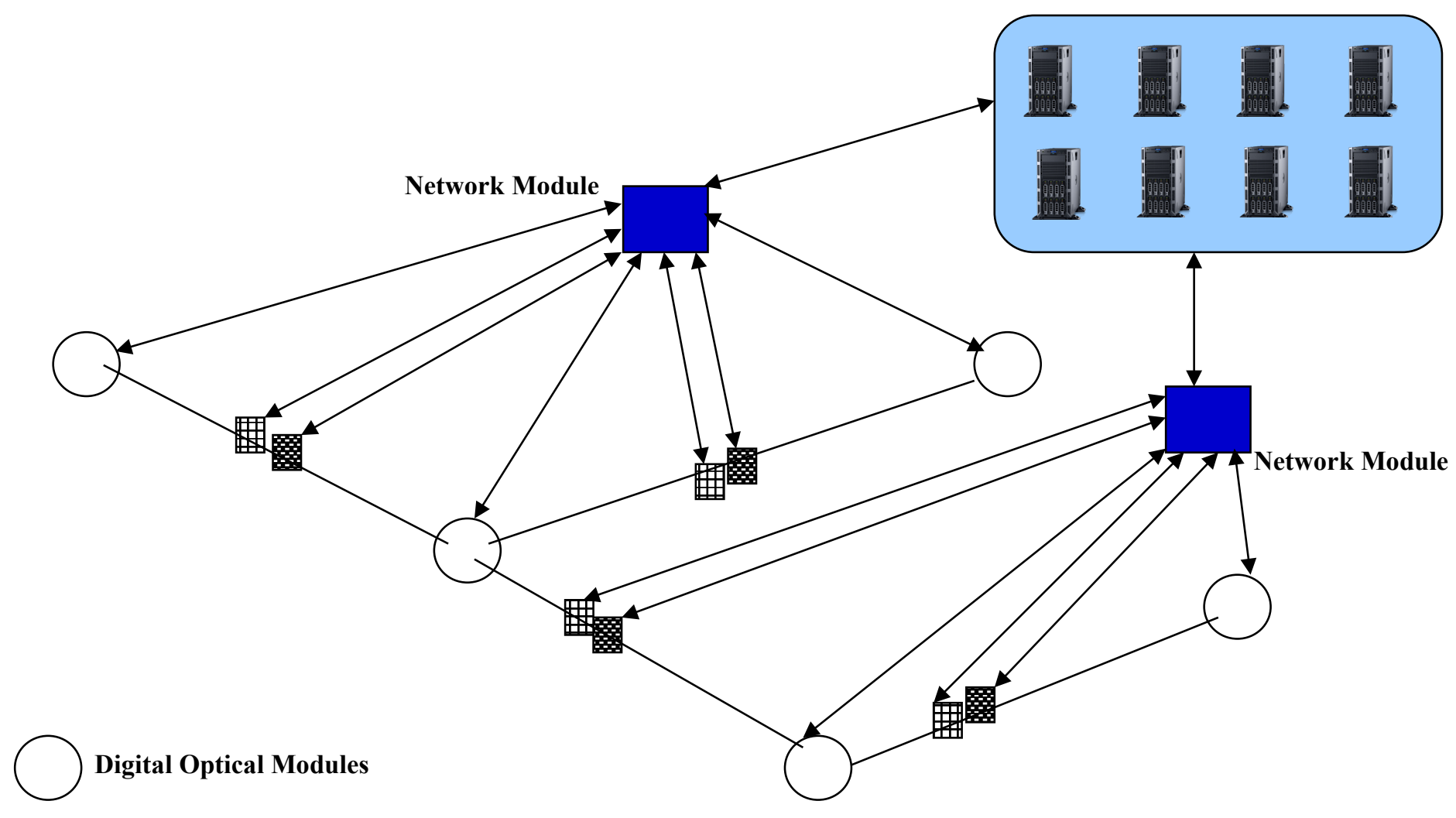

Underwater Video Camera

睪 Underwater Acoustic Camera

Figure 1: Network Scenario showing the role of the Network Module and Onshore Computing Entity 
some cameras and sensors are compensated by cameras and sensors with improved capabilities. Viewing diversity implies the ability of either an acoustic or video camera being able to detect objects that cannot be detected by another acoustic or video camera. This is feasible considering the heterogeneous capabilities of sensors and cameras in a large spatial underwater network.

The consideration of viewing diversity reduces the need to explicitly consider the detecting ranges of sensors and the cameras being presented in Figure 1. This reduces underwater surveillance network design complexity because additional details on the individual technical capabilities of each underwater acoustic sensor and underwater video camera need not be individually considered. In Figure 1, the underwater camera sensors and underwater acoustic camera are stationary. The underwater camera sensors and underwater acoustic cameras differ from the underwater telescope. The underwater telescope is stationary, executes the functionality of the scientific underwater vehicle and is realized by the digital optical module.

As seen in Figure 1, the deployment of the underwater telescopes is considered at different ocean depths. Each depth is also associated with a distance from a reference shore. The underwater cameras and sensors are integrated in the string gaps existing between underwater telescopes. Hence, they constitute the sensors that are placed in the string gaps. String gaps are the spaces un-utilised between conductors that link the digital optical modules intended to capture neutrino radiation in underwater astronomy observation.

Therefore, the sensors placed in the string gaps are also deployed at different ocean depths. The data on the ocean depths are acquired and accessed while planning for the deployment of the underwater telescopes. The underwater cameras and sensors acquire information from a three dimensional perspective. The considered dimensions are the: (1) concerned ocean depth, (2) distance from the reference shore and (3) Epoch of observation. The information on the concerned ocean depth, distance from the reference shore and epoch of observation are associated with each observation by the concerned sensor or camera.

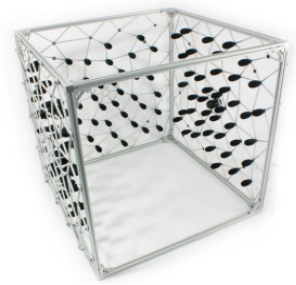

Figure 2: An acoustic camera capable of underwater operation [34]

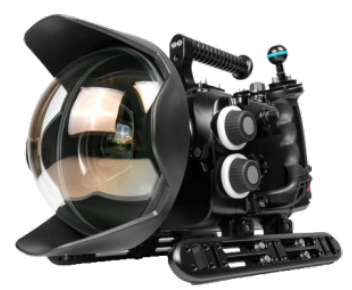

Figure 3: An underwater video camera suitable for proposed use [35]

The illustration in Figure 4 shows the execution of observation by the underwater acoustic camera and underwater video camera in the string gap. Figure 4 shows the three dimensional representation as seen in the varying distances of the considered regions from a reference shore and the differing depths. Each region of the underwater environment is being observed by the digital optical module. The combination of each region alongside the underwater acoustic camera and underwater video camera is known as the logical observation group. In Figure 4, there are four logical observation groups i.e. logical observation group 1, logical observation group 2, logical observation group 3 and logical observation group 4. In each observation group, underwater acoustic cameras and underwater video cameras that are integrated into the string gap observe the concerned area. The observation is accompanied with data recording alongside the information on the depth (distance along the vertical i.e., $y$-axis), distance from the reference shore (along the $\mathrm{x}$-axis) at different epochs (being the time dimension). An observation in this manner results in a threedimensional observation.

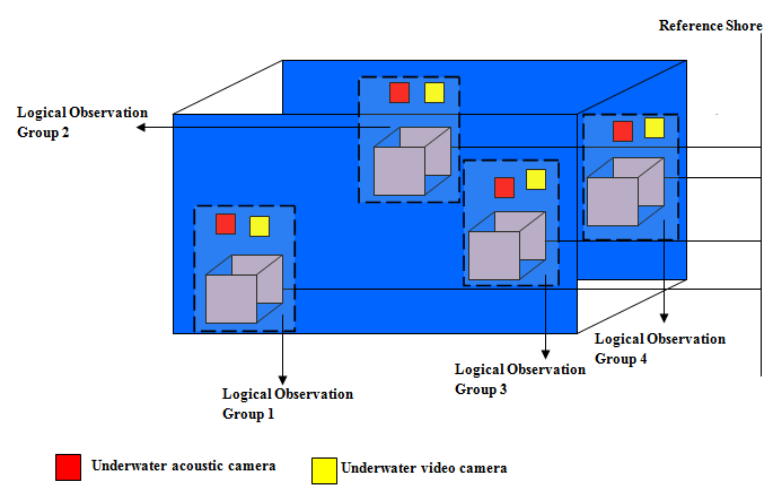

Figure 4: Scenario describing the role of underwater acoustic camera and underwater video camera in observing selected area of the underwater environment. These sensors are integrated into the string gap.

\section{Underwater Astronomy - Knowledge Advances and Benefits to Computing Systems Design}

The discussion in this section describes how the conduct of underwater astronomy enhances knowledge advances in near ocean and underwater computing systems design. The underwater environment hosts a significant number of applications besides underwater astronomy such as networking and computing. This section has two parts. The first describes the rationale for considering the impact of underwater astronomy in computing networks. The second presents the supporting system model.

\subsection{Underwater Astronomy and Computing Systems - A Rationale}

The emergence of underwater astronomy necessitates the design of onshore computing station networks. The processing of data arising from underwater astronomy requires designing onshore computing station networks. This is capital intensive. Therefore, it is important to design solutions that reduce the associated costs. The ability of underwater astronomy's computing station to potentially address this challenge is considered in this aspect.

\subsection{Underwater Astronomy and Computing - Architecture and System Design Concerns}

The advent of underwater astronomy increases the amount of ocean data requiring processing. This increases the volume of data 
requiring access to computing resources for processing. The need to enable data processing has necessitated the deployment of onshore computing stations to receive and process data from underwater neutrino telescopes [36-37]. In [36], the trigger and data acquisition system is not integrated with cloud computing systems. Therefore, organizations seeking to deploy underwater telescopes require ownership of onshore computing platforms. The drawback to this perspective is that it increases the cost of conducting underwater astronomy. This is because all organizations desiring to conduct underwater astronomy have to deploy own computing platforms and do not subscribe to existing terrestrial cloud computing platforms. Therefore, it is desirable to design novel network architecture linking onshore computing entities to existing cloud computing platforms. This is because cloud computing has been recognized to be beneficial to astronomy [37]. In the case of underwater astronomy, underwater data centres such as those in [38] are useful. This contributes to knowledge in the area of wireless network architecture.

The use of underwater computing platforms also enhances the PUE in comparison to terrestrial computing platforms [39-40]. The use of underwater computing platforms in data processing is suitable for processing scientific data as seen in [41]. However, designing architecture and network enabling the underwater astronomy telescopes to utilize underwater data centres requires research attention. The need to integrate underwater telescope data processing with underwater computing platforms is beneficial in two aspects. The first is that it enables the design of integrated systems that connect underwater telescope arrays with underwater computing platforms. This is advantageous in terms of contributing to knowledge in systems architecture. The second is that the use of underwater computing platforms opens a new vista for sub-marine application data processing. Therefore, underwater astronomy has prospects in advancing research in network architecture and integrated systems.

\section{Underwater Astronomy - Enabling Underwater Surveillance}

Deploying underwater telescopes over a vast sub-ocean area provides vast ocean coverage thereby enabling the development of an underwater surveillance strategy. The resulting large area coverage provides a potential underwater surveillance application. This enables the realization of an underwater surveillance network. The discussion here has two aspects. The first discusses the rationale for considering how underwater astronomy can enable underwater surveillance. The second presents a potential system model.

\subsection{Underwater Astronomy and Surveillance Applications - Rationale}

The deployment and maintenance of underwater telescopes necessitates and increases human-ocean interaction. The maintenance of underwater telescopes can be done at the ocean shore or aboard an ocean vessel at the expense of high logistics costs; and reduced underwater telescope observation time. The maintenance can also be conducted in the underwater environment with reduced degradation in the observation ability of other underwater telescopes. However, this is at the expense of hiring ocean divers for the execution of maintenance procedures. This is challenging because telescope engineers and technicians are not trained ocean divers. Alternatively, submarines can also be used at the expense of high costs. Nevertheless, these approaches facilitate human-ocean interaction which is useful for underwater surveillance. Underwater surveillance approaches are considered from two perspectives. The first and second considers that maintenance of underwater telescopes is done on the surface (shore or aboard an ocean vessel) and in the underwater environment, respectively.

\subsection{Underwater Astronomy-Architecture and System Design Concerns}

Underwater surveillance and maritime security are closely related [42-43]. Underwater security can be realized via the use of submarines or autonomous underwater vehicles which is capital intensive. The high cost makes it challenging to ensure adequate underwater maritime security. A conservative estimate shows that a sum of about $\$ 20 \mathrm{~B}$ is required to design and realize an operational submarine [44]. This is beyond the reach of capital constrained organizations.

Therefore, developing nations have challenges in using submarines for underwater surveillance and security. Autonomous underwater vehicles are a feasible low-cost alternative to submarines. However, deploying multiple autonomous underwater vehicles also has high costs. The scenario shown in Figure 1 describes how cameras can be integrated in the string gap of underwater telescopes. These cameras can be integrated during the execution of a scheduled telescope maintenance manoeuvre.

The deployment of underwater telescopes alongside underwater cameras is suitable for realizing underwater surveillance. In this case, the underwater cameras give a video output that is processed by the organization(s) deploying the underwater cameras and owns the copyright to the video content. The resulting video is processed to extract security related information from the received underwater data. The network of video cameras is also suited for underwater surveillance and is cheaper than using multiple autonomous underwater vehicles. The realization of an underwater surveillance network enables advances in: (i) Video acquisition via underwater camera networks, (ii) Video processing with focus on preference of underwater surveillance, and (iii) integration of video processing results with cloud computing platforms.

Underwater surveillance can also be addressed when the maintenance is conducted in the underwater environment. The design of an underwater vehicle with lower acquisition and operational costs in comparison to submarines is proposed. The required capability can be realized via variable duration short mission manned underwater vehicles. The proposed variable duration short mission underwater vehicle serves as an underwater habitat that hosts telescope technicians and engineers. It hosts the payload used to execute the maintenance procedures. The variable duration short mission underwater vehicle operates for a maximum of 3 hours in the underwater environment before returning to the surface/shore. The telescope technicians and engineers execute the maintenance via robotic arms and do not exit the variable duration short mission underwater vehicle's interior.

The proposed variable duration short mission underwater vehicle is suitable for use by naval authorities for underwater 
surveillance applications. In this case, the robotic arms host camera payload and enables the operator to record image and video of the underwater environment. Therefore, regional and national organizations such as the Navy can benefit from technological advancements arising from the need to conduct underwater astronomy.

\section{Value Addition - Aspects of the Blue Economy}

The development of the blue economy enhances future economic growth $[45,46]$ and can be realized via marine spatial planning [47, 48]. Marine spatial planning determines the suitability of marine resources for use in different ocean applications. The integration of sensors in the 'string gap' makes it easy to monitor the ocean. The acquired data can be used to determine the best way for allocating ocean resources.

The locating of multiple underwater telescopes in the ocean requires the conduct of ocean survey $[47,48]$. This enables the determination of best ocean zones to site underwater telescopes. For example, it is noted in [49] that water composition influences the variation of water's optical parameters. This implies that ocean site selection for placing underwater telescopes involves the acquisition of knowledge on sea water's instantaneous optical and chemical properties. The chemical properties of water influence other factors such as the time varying underwater specific heat capacity. This is useful in the determination of the suitable location for siting underwater data centres.

The discussion in [50] notes that the exploration of maritime resources such as Lake Baikal (Domogatsky, Bezrukov), Atlantic Ocean, Black Sea, Indian Ocean, Pacific Ocean and Mediterranean Ocean (with sites in Zheleznyk and Petrukhin). The exploration of maritime resources in these locations enables the acquisition of data on the maritime resources at the mentioned locations. The acquired underwater maritime underwater data provides a significant amount of underwater and oceanographic big data. The data is suitable for use in determining the physical properties of different aspects of the underwater environment at different epochs. The role of such data in marine spatial planning (for different ocean zones) is recognized in $[51,52]$.

The blue economy encompasses a significant number of opportunities such as seabed mining [53], large scale fishing, maritime transportation, underwater tourism and seaweed farming. The conduct of underwater astronomy observations provides an opportunity to explore the sub-surface ocean environment. This makes it feasible to determine underwater ocean zones suitable for deploying the applications identified in $[54,55]$.

The prospects and challenges of underwater technological development has motivated by the conduct of underwater astronomy is shown in Table I. Table I focuses on the prospects and benefits associated with underwater surveillance and the blue economy. The information presented in Table I focuses on how the desire to conduct underwater astronomy can make contributions to underwater surveillance and the blue economy.

\section{Performance Formulation}

This section examines the benefit of the proposed solution and has two parts. The first and second aspects discuss the performance benefits and formulate the performance model, respectively.
Table 1: Potential Benefits to Ocean Surveillance, Underwater Security and Blue Economy

\begin{tabular}{|c|c|c|}
\hline $\mathrm{S} / \mathrm{N}$ & Sector & Potential Contributions \\
\hline 1 & $\begin{array}{l}\text { Underwater } \\
\text { Security } \\
\text { (infrastructure } \\
\text { Acquisition) }\end{array}$ & $\begin{array}{l}\text { Variable duration short mission manned } \\
\text { underwater vehicles can be used as low } \\
\text { cost entities with capabilities similar to a } \\
\text { submarine and suitable for short length } \\
\text { missions by a Naval force. }\end{array}$ \\
\hline 2 & $\begin{array}{ll}\text { Naval } & \text { Security } \\
\text { Services } & \end{array}$ & $\begin{array}{l}\text { The use of cameras integrated in the } \\
\text { string gap for underwater surveillance } \\
\text { improves the naval surveillance services } \\
\text { because of the use of a distributed } \\
\text { underwater camera network. }\end{array}$ \\
\hline 3 & $\begin{array}{l}\text { Technological } \\
\text { Development- } \\
\text { Robotics }\end{array}$ & $\begin{array}{l}\text { The need to design robotic arms for } \\
\text { underwater maintenance and camera } \\
\text { positioning contributes to research and } \\
\text { technological development. }\end{array}$ \\
\hline 4 & $\begin{array}{l}\text { Astronomy } \\
\text { Organizations }\end{array}$ & $\begin{array}{l}\text { Sharing of onshore computing facility } \\
\text { costs with Naval organizations reduces } \\
\text { the cost burden on astronomy } \\
\text { organizations. }\end{array}$ \\
\hline 5 & $\begin{array}{l}\text { Entertainment: } \\
\text { Underwater Media }\end{array}$ & $\begin{array}{l}\text { Acquired underwater video can be sold } \\
\text { to channels where it can be viewed as } \\
\text { entertaining video content. }\end{array}$ \\
\hline 6 & $\begin{array}{ll}\text { Marine } & \text { Spatial } \\
\text { Planning } & \end{array}$ & $\begin{array}{l}\text { The obtained images and video of the } \\
\text { underwater environment can be used to } \\
\text { characterize the ocean environment. } \\
\text { This is useful for marine spatial planning } \\
\text { purposes }\end{array}$ \\
\hline 7 & $\begin{array}{l}\text { Blue Economy- } \\
\text { Application } \\
\text { Identification }\end{array}$ & $\begin{array}{l}\text { A successful marine spatial planning } \\
\text { process helps in determining suitable } \\
\text { revenue earning applications that can be } \\
\text { hosted in a given ocean zones. }\end{array}$ \\
\hline 8 & $\begin{array}{l}\text { Knowledge } \\
\text { Contribution }- \\
\text { Chemical and } \\
\text { Optical Properties }\end{array}$ & $\begin{array}{l}\text { The study of the optical properties of } \\
\text { ocean water for determining the viability } \\
\text { of an underwater location for hosting } \\
\text { telescopes provides opportunity to } \\
\text { understand the relations between } \\
\text { chemical composition of water and its } \\
\text { optical properties at an epoch. }\end{array}$ \\
\hline 9 & $\begin{array}{l}\text { Computing-Future } \\
\text { Computing } \\
\text { Platforms }\end{array}$ & $\begin{array}{l}\text { The acquisition of information on } \\
\text { chemical properties is suitable for } \\
\text { determining the viability of a given } \\
\text { maritime resources for hosting } \\
\text { underwater data centres; determining } \\
\text { number of suitable hosting locations. }\end{array}$ \\
\hline 10 & $\begin{array}{l}\text { Knowledge } \\
\text { Contribution- } \\
\text { Ocean Maps }\end{array}$ & $\begin{array}{l}\text { The information obtained via cameras } \\
\text { and used to develop a profile of different } \\
\text { underwater ocean zones can be used to } \\
\text { develop an ocean profile or underwater } \\
\text { map. This is a novel contribution to } \\
\text { knowledge. }\end{array}$ \\
\hline 11 & $\begin{array}{l}\text { Human Capital } \\
\text { Development-- } \\
\text { Naval } \\
\text { Infrastructure }\end{array}$ & $\begin{array}{l}\text { The development of variable length } \\
\text { duration manned underwater vehicles } \\
\text { (with submarine capability) enables the } \\
\text { development of human capital in the } \\
\text { aspect of underwater vehicle hardware } \\
\text { design and manufacture; as well as } \\
\text { software design and realization. }\end{array}$ \\
\hline 12 & $\begin{array}{l}\text { Human Capital } \\
\text { Development-- } \\
\text { Software }\end{array}$ & $\begin{array}{l}\text { Processing of Video from underwater } \\
\text { environment for security preferences } \\
\text { enables skill acquisition with regard to } \\
\text { video editing software design and } \\
\text { development. }\end{array}$ \\
\hline
\end{tabular}




\subsection{Performance Benefits}

The discussion has proposed the adoption of a multi-domain approach to ocean exploration with a focus on underwater astronomy. In the proposed consideration, external technological and scientific interests are recognized to benefit from the conduct of underwater astronomy. The resulting crowd-sourcing effort reduces the costs of conducting underwater astronomy. In addition, the launch of multiple underwater telescope units' results in an array that enhances the resolution of underwater astronomy observations. This is because multiple entities that were previously unconsidered now launch additional logical underwater telescope units. The benefit of this is an increase in the baseline of the resulting underwater interferometer system. From a perspective of general observation, a longer baseline is beneficial to obtain improved resolution in astronomy observations [56-57]. The launch of additional logical underwater telescope unit at low costs due to the incorporation of a crowd-sourced approach increases the interferometer size and baseline. This improves the observation resolution of underwater astronomy systems.

The integration of onshore computing platforms into the existing cloud computing platform framework increases the computational resources accessible to computing applications. Two computing applications are recognized in this regard. These are underwater astronomy and wireless computing applications. In addition, onshore computing platforms are located at vantage locations where they can easily benefit from free cold air cooling. This is because onshore computing platforms are able to take advantage of the cold air and cold water available in nearby maritime resources such as rivers and the ocean shore. This reduces the reliance on the use of conventional cooling methods that uses components such as air conditioners, pumps and chillers. Therefore, the integration of onshore computing platforms into existing data centers can enhance the PUE.

Therefore, the proposed multi-domain perspective has three benefits. These are: (i) increasing the computational resources accessible to underwater astronomy, wireless networks and computing applications, (ii) Enhancing the PUE of existing cloud computing platforms and (iii) improving the resolution of underwater astronomy.

\subsection{Performance Formulation}

Let $\alpha$ and $\beta$ denote the set of vessels and cameras (integrated in the string gap), respectively.

$$
\begin{aligned}
& \alpha=\left\{\alpha_{1}, \alpha_{2}, \ldots, \alpha_{A}\right\} \\
& \beta=\left\{\beta_{1}, \beta_{2}, \ldots, \beta_{B}\right\}
\end{aligned}
$$

In addition, let $\gamma$ and ' $\Upsilon$ be the set of onshore computing platforms and data centers (cloud computing platforms), respectively.

$$
\begin{aligned}
& \gamma=\left\{\gamma_{1}, \gamma_{2}, \ldots, \gamma_{C}\right\} \\
& \Upsilon=\left\{\Upsilon_{1}, \Upsilon_{2}, \ldots, \Upsilon_{D}\right\}
\end{aligned}
$$

The set of servers associated with the onshore computing platform $\gamma_{c}, \gamma_{c} \in \gamma$ and data centers (cloud computing platforms) $\Upsilon_{\mathrm{d}}, \Upsilon_{\mathrm{d}} \in \Upsilon^{\prime} \Upsilon$ are given as:

$$
\begin{gathered}
\gamma_{\mathrm{c}}=\left\{\gamma_{\mathrm{c}}^{1}, \gamma_{\mathrm{c}}^{2}, \ldots, \gamma_{\mathrm{c}}^{\mathrm{I}}\right\} \\
\Upsilon^{\prime} \Upsilon_{\mathrm{d}}=\left\{\Upsilon^{\prime} \Upsilon^{\prime} \Upsilon_{\mathrm{d}}^{1}, \Upsilon^{\prime} \Upsilon_{\mathrm{d}}^{2}, \ldots, \Upsilon^{\prime} \Upsilon_{\mathrm{d}}^{\mathrm{J}}\right\}
\end{gathered}
$$

Let $\mathrm{C}_{2}\left(\gamma_{\mathrm{c}}^{\mathrm{i}}, \mathrm{t}_{\mathrm{y}}\right), \gamma_{\mathrm{c}}^{\mathrm{i}} \in \gamma_{\mathrm{c}}, \mathrm{t}_{\mathrm{y}} \in \mathrm{t}$ denote the computational resources accessible on $\mathrm{i}^{\text {th }}$ server aboard the $\mathrm{c}^{\text {th }}$ onshore computing platform $\gamma_{\mathrm{c}}^{\mathrm{i}}$ at the $\mathrm{y}^{\text {th }}$ epoch $\mathrm{t}_{\mathrm{y}}$. The computational resources accessible on the $\mathrm{j}^{\text {th }}$ server aboard the $\mathrm{d}^{\text {th }}$ computing platform $\Upsilon_{d}^{j}, \Upsilon_{d}^{j} \in \Upsilon_{d}, t_{y} \in t$ at the $y^{\text {th }}$ epoch $t_{y}$ is denoted $C_{2}\left(\Upsilon_{d}^{j}, t_{y}\right)$. In the case, where cloud computing platforms are not accessed by underwater astronomy applications, the accessible computing resources, $\theta_{1}$ is

$$
\theta_{1}=\sum_{\mathrm{c}=1}^{\mathrm{C}} \sum_{\mathrm{i}=1}^{\mathrm{I}} \sum_{\mathrm{y}=1}^{\mathrm{Y}} \mathrm{C}_{2}\left(\gamma_{\mathrm{c}}^{\mathrm{i}}, \mathrm{t}_{\mathrm{y}}\right)
$$

In the case where cloud computing platforms are accessible by underwater astronomy applications, the accessible computing resources, $\theta_{2}$ is:

$$
\theta_{2}=\sum_{\mathrm{c}=1}^{\mathrm{C}} \sum_{\mathrm{i}=1}^{\mathrm{I}} \sum_{\mathrm{y}=1}^{\mathrm{Y}} \mathrm{C}_{2}\left(\gamma_{\mathrm{c}}^{\mathrm{i}}, \mathrm{t}_{\mathrm{y}}\right)+\sum_{\mathrm{d}=1}^{\mathrm{D}} \sum_{\mathrm{j}=1}^{\mathrm{J}} \sum_{\mathrm{y}=1}^{\mathrm{Y}} \mathrm{C}_{2}\left({ }^{\prime} \mathrm{Y}_{\mathrm{d}}^{\mathrm{j}}, \mathrm{t}_{\mathrm{y}}\right)
$$

The power required to operate the servers $\gamma_{c}^{i}$ and ${ }^{\prime} \Upsilon_{d}^{j}$ at the epoch $t_{y}$ are denoted as $P_{1}\left(\gamma_{c}^{i}, t_{y}\right)$ and $P_{1}\left({ }^{j}{ }_{d}^{j}, t_{y}\right)$, respectively. In addition, the power required to cool the servers $\gamma_{c}^{i}$ and ${ }^{\prime} \Upsilon_{d}^{j}$ at the epoch $\mathrm{t}_{\mathrm{y}}$ are denoted as $\mathrm{P}_{2}\left(\gamma_{\mathrm{c}}^{\mathrm{i}}, \mathrm{t}_{\mathrm{y}}\right)$ and $\mathrm{P}_{2}\left({ }^{\mathrm{j}} \mathrm{d}, \mathrm{t}_{\mathrm{y}}\right)$, respectively. The PUE of a cloud computing platform comprising only servers in existing data centers $v_{1}$ is:

$$
v_{1}=\sum_{\mathrm{c}=1}^{\mathrm{C}} \sum_{\mathrm{i}=1}^{\mathrm{I}} \sum_{\mathrm{y}=1}^{\mathrm{Y}}\left(\frac{\mathrm{P}_{1}\left(\gamma_{\mathrm{c}}^{\mathrm{i}}, \mathrm{t}_{\mathrm{y}}\right)+\mathrm{P}_{2}\left(\gamma_{\mathrm{c}}^{\mathrm{i}}, \mathrm{t}_{\mathrm{y}}\right)}{\mathrm{P}_{1}\left(\gamma_{\mathrm{c}}^{\mathrm{i}}, \mathrm{t}_{\mathrm{y}}\right)}\right)
$$

When the cloud platform comprises both non-onshore based servers and onshore based servers, the PUE of the resulting cloud computing platform, $v_{2}$ is:

$$
\begin{gathered}
v_{2}=\sum_{c=1}^{C} \sum_{d=1}^{D} \sum_{i=1}^{I} \sum_{j=1}^{J}\left(\sum_{y=1}^{Y} \frac{A+B}{P_{1}\left(\gamma_{c}^{i}, t_{y}\right)+P_{1}\left(\gamma^{\prime} Y_{d}^{j}, t_{y}\right)}\right) \\
A=P_{1}\left(\gamma_{c}^{i}, t_{y}\right)+P_{1}\left(\gamma^{\prime} Y_{d}^{j}, t_{y}\right) \\
B=P_{2}\left(\gamma_{c}^{i}, t_{y}\right)+\left(1-\Gamma\left(j, d, t_{y}\right)\right) P_{2}\left(\gamma^{\prime} Y_{d}^{j}, t_{y}\right)
\end{gathered}
$$

$\Gamma\left(\mathrm{j}, \mathrm{d}, \mathrm{t}_{\mathrm{y}}\right)$ is the reduction in the cooling energy on the server ${ }^{\prime} \Upsilon_{\mathrm{d}}^{\mathrm{j}}$ due to the maritime resource at the $y^{\text {th }}$ epoch $t_{y}$.

Furthermore, let $\zeta$ denote the set of organizations that benefit from the proposed integration approach such that:

$$
\begin{gathered}
\zeta=\left\{\zeta_{\mathrm{ao}}, \zeta_{\text {nao }}\right\} \\
\zeta_{\mathrm{ao}}=\left\{\zeta_{\mathrm{aoo}}^{1}, \zeta_{\mathrm{ao}}^{2}, \ldots, \zeta_{\mathrm{ao}}^{\mathrm{F}}\right\} \\
\zeta_{\text {nao }}=\left\{\zeta_{\text {nao }}^{1}, \zeta_{\text {nao }}^{2}, \ldots, \zeta_{\text {nao }}^{\mathrm{M}}\right\}
\end{gathered}
$$

$\zeta_{\text {ao }}$ and $\zeta_{\text {nao }}$ are the set of underwater astronomy organizations and non-underwater astronomy organizations respectively.

$\zeta_{\mathrm{ao}}^{\mathrm{f}}, \zeta_{\mathrm{ao}}^{\mathrm{f}} \in \zeta_{\mathrm{ao}}$ and $\zeta_{\text {nao }}^{\mathrm{m}}, \zeta_{\text {nao }}^{\mathrm{m}} \in \zeta_{\text {nao }}$ are the $\mathrm{f}^{\text {th }}$ underwater astronomy organization and $\mathrm{m}^{\text {th }}$ non-underwater astronomy organization, respectively. 
The underwater telescopes associated with the $\mathrm{f}^{\text {th }}$ underwater astronomy and $\mathrm{m}^{\text {th }}$ non-underwater astronomy organizations are given as:

$$
\begin{array}{r}
\zeta_{\mathrm{ao}}^{\mathrm{f}}=\left\{\zeta_{\mathrm{ao}}^{\mathrm{f}, 1}, \zeta_{\mathrm{ao}}^{\mathrm{f}, 2}, \ldots, \zeta_{\mathrm{ao}}^{\mathrm{f}, \mathrm{Q}}\right\} \\
\zeta_{\text {nao }}^{\mathrm{m}}=\left\{\zeta_{\text {nao }}^{\mathrm{m}, 1}, \zeta_{\text {nao }}^{\mathrm{m}, 2}, \ldots, \zeta_{\text {nao }}^{\mathrm{m}, \mathrm{L}}\right\}
\end{array}
$$

The baseline for underwater telescopes $\zeta_{\mathrm{ao}}^{\mathrm{f}, \mathrm{q}-1}, \zeta_{\text {ao }}^{\mathrm{f}, \mathrm{q}-1} \in \zeta_{\mathrm{ao}}^{\mathrm{f}},(\mathrm{q}-$ $1) \geq 1$ (the $(q-1)^{\text {th }}$ underwater telescope belonging to the underwater astronomy organization) and $\zeta_{\text {ao }}^{\mathrm{f}, \mathrm{q}}, \zeta_{\text {ao }}^{\mathrm{f}, \mathrm{q}} \in \zeta_{\text {ao }}^{\mathrm{f}}$ (the $(q)^{\text {th }}$ underwater telescope belonging to the underwater astronomy organization) at the epoch $\mathrm{t}_{\mathrm{y}}$ is denoted $\ddot{\mathrm{r}}\left(\zeta_{\mathrm{ao}}^{\mathrm{f}, \mathrm{q}-1}, \zeta_{\mathrm{ao}}^{\mathrm{f}, \mathrm{q}}, \mathrm{t}_{\mathrm{y}}\right)$.

The underwater interferometer baseline without and with the synergy are $B_{1}$ and $B_{2}$, respectively and given as:

$$
\begin{gathered}
\mathrm{B}_{1}=\sum_{\mathrm{y}=1}^{\mathrm{Y}}\left(\sum_{\mathrm{f}=1}^{\mathrm{F}} \sum_{\mathrm{q}=2}^{\mathrm{Q}} \ddot{\mathrm{Y}}\left(\zeta_{\mathrm{ao}}^{\mathrm{f}, \mathrm{q}-1}, \zeta_{\mathrm{ao}}^{\mathrm{f}, \mathrm{q}}, \mathrm{t}_{\mathrm{y}}\right)\right) \\
B_{2}=\sum_{y=1}^{Y}(C+D) \\
C=\sum_{f=1}^{F} \sum_{q=2}^{Q} \ddot{Y}\left(\zeta_{a o}^{f, q-1}, \zeta_{a o}^{f, q}, t_{y}\right) \\
D=\sum_{m=1}^{M} \sum_{l=2}^{L} \ddot{Y}\left(\zeta_{\text {nao }}^{m, l-1}, \zeta_{\text {nao }}^{m, l}, t_{y}\right)
\end{gathered}
$$

The resolution of the underwater astronomy radiation if $\lambda$ is the Cherenkov radiation wavelength, without and with the proposed synergy is denoted $f_{1}$ and $f_{2}$, respectively:

$$
f_{e}=\frac{\lambda}{B_{e}}, e \in\{1,2\}
$$

\section{Performance Evaluation and Analysis}

The performance evaluation of the proposed mechanism is done using the parameters in Table 2. The computational resource aboard the servers used in the simulation has been chosen considering the parameters used in [58]. The proportion of energy used in cooling servers aboard terrestrial data centers is considered to have a value of $40 \%$ as seen in [59]. The value of the power consumption used in servers is motivated by information obtained from [60]. In Table 2, the coverage of underwater telescopes from participating organizations differs from the coverage of telescopes deployed by underwater astronomy organizations. In addition, the baseline in both cases has been selected to have a similar range of values. This is done to prevent a scenario where a greedy approach is used when adding underwater telescopes from participating organizations. The performance simulation and analysis has been performed with the MATLAB software package.

The results for the simulated accessible computational resources and PUE performance is shown in Figure 5 and Figure 6 , respectively. The results obtained and presented in Figure 5 www.astesj.com shows that the joint usage of onshore computing platforms alongside existing terrestrial computing platforms increases the accessible computational resources. In the existing case, a server unit is a server in an existing terrestrial computing platform. A server unit in the proposed case is a logical combination of individual servers in the onshore and existing terrestrial computing platforms.

Figure 6 shows that the use of the onshore computing platforms with the existing terrestrial computing platforms improves the PUE (a lower PUE is better) because of the reduced cooling energy in onshore computing platforms as they are closer to maritime resources. Onshore computing platforms are sited in near long term low temperature environments such as rural maritime resources. This increases the available cold water that can be freely accessed for server cooling. Such a performance benefit can be seen in the Google Hamina onshore computing platform [60].

Analysis of the results shows that leveraging on a synergy between underwater astronomy organizations and identified technology interests enhance the accessible computational resources, and the PUE. The accessible computational resources and PUE are enhanced by an average of $48.8 \%$ and $1.6 \%$, respectively.

The influence of the proposed synergy on the observation resolution is also investigated and the obtained result is in Figure 7. Figure 7 shows that observation resolution improves with an increase in the number of logical telescope units. A lower observation resolution implies that more details can be obtained from the observation procedure and is better than a higher observation resolution. In the proposed case, a telescope deployed by a participating organization and the one telescope deployed by the underwater astronomy organization comprises a logical telescope. However, the logical telescope unit has a longer baseline (i.e. telescope separation) thereby resulting in a lower observation resolution. The simulation uses an observation wavelength of $450 \mathrm{~nm}$ for the Cherenkov radiation as obtained from [61]. Analysis shows that the synergy enhances the observation resolution by $41.3 \%$ on average.

Table 2: Parameters used for Performance Evaluation

\begin{tabular}{|l|l|l|}
\hline S/N & \multicolumn{1}{|c|}{ Parameter } & \multicolumn{1}{|c|}{ Value } \\
\hline 1 & $\begin{array}{l}\text { Quantity of Servers in the Onshore } \\
\text { Computing Platform }\end{array}$ & 70 \\
\hline 2 & $\begin{array}{l}\text { Quantity of Servers in the Existing } \\
\text { Terrestrial Computing Platform }\end{array}$ & 120 \\
\hline 3 & $\begin{array}{l}\text { Minimum Computational Resources in the } \\
\text { Onshore and Existing Terrestrial } \\
\text { Computing Platform Server }\end{array}$ & $\begin{array}{l}{[2.02,} \\
\text { Gbytes }\end{array}$ \\
\hline 4 & $\begin{array}{l}\text { Maximum Computational Resources in the } \\
\text { Onshore and Existing Terrestrial Computing } \\
\text { Platform Server }\end{array}$ & $\begin{array}{l}{[78.75,95.95]} \\
\text { Gbytes }\end{array}$ \\
\hline 5 & $\begin{array}{l}\text { Average Computational Resources in the } \\
\text { Onshore and Existing Terrestrial Computing } \\
\text { Platform Server }\end{array}$ & $\begin{array}{l}{[35.47,44.52]} \\
\text { Gbytes }\end{array}$ \\
\hline 6 & $\begin{array}{l}\text { Minimum Power Consumed by Server in the } \\
\text { Onshore and Existing Terrestrial Computing } \\
\text { Platform Server }\end{array}$ & {$[0.38,1.48] \mathrm{W}$} \\
\hline 7 & $\begin{array}{l}\text { Maximum Power Consumed by Server in } \\
\text { the Onshore and Existing Terrestrial } \\
\text { Computing Platform Server }\end{array}$ & {$[46.29, \quad 71.78]$} \\
$\mathrm{W}$
\end{tabular}




\begin{tabular}{|l|l|l|}
\hline 8 & $\begin{array}{l}\text { Average Power Consumed by Server in the } \\
\text { Onshore and Existing Terrestrial } \\
\text { Computing Platform Server }\end{array}$ & $\mathrm{W}$ \\
\hline 9 & $\begin{array}{l}\text { Proportion of power used in cooling servers } \\
\text { in existing terrestrial data centers }\end{array}$ & $40 \%$ \\
\hline 10 & $\begin{array}{l}\text { Minimum proportion of cooling power } \\
\text { reduction due to onshore location }\end{array}$ & $0.1 \%$ \\
\hline 11 & $\begin{array}{l}\text { Maximum proportion of cooling power } \\
\text { reduction due to onshore location }\end{array}$ & $4.92 \%$ \\
\hline 12 & $\begin{array}{l}\text { Average proportion of cooling power } \\
\text { reduction due to onshore location }\end{array}$ & $2.54 \%$ \\
\hline 13 & $\begin{array}{l}\text { Number of Underwater Astronomy } \\
\text { Organizations }\end{array}$ & 1 \\
\hline 14 & Number of Participating Organizations & 1 \\
\hline 15 & $\begin{array}{l}\text { Minimum Baseline of Underwater } \\
\text { Telescopes from Underwater Astronomy } \\
\text { Organizations }\end{array}$ & $9.50 \mathrm{~m}$ \\
\hline 16 & $\begin{array}{l}\text { Maximum Baseline of Underwater } \\
\text { Telescopes from Underwater Astronomy } \\
\text { Organizations }\end{array}$ & $1.99 \mathrm{~km}$ \\
\hline 17 & $\begin{array}{l}\text { Average Baseline of Underwater Telescopes } \\
\text { from Underwater Astronomy Organizations }\end{array}$ & $1.01 \mathrm{~km}$ \\
\hline 18 & $\begin{array}{l}\text { Minimum Baseline of Underwater } \\
\text { Telescopes from Participating Organizations }\end{array}$ & $114.98 \mathrm{~m}$ \\
\hline 19 & $\begin{array}{l}\text { Maximum Baseline of Underwater } \\
\text { Telescopes from Participating Organizations }\end{array}$ & $1.43 \mathrm{~km}$ \\
\hline 20 & $\begin{array}{l}\text { Average Baseline of Underwater Telescopes } \\
\text { from Participating Organizations }\end{array}$ & $0.79 \mathrm{~km}$ \\
\hline 21 & $\begin{array}{l}\text { Wavelength of the Cherenkov Radiation } \\
\text { under observation ( [61]) }\end{array}$ & $450 \mathrm{~nm}$. \\
\hline & \multicolumn{1}{|c|}{. } \\
\hline
\end{tabular}

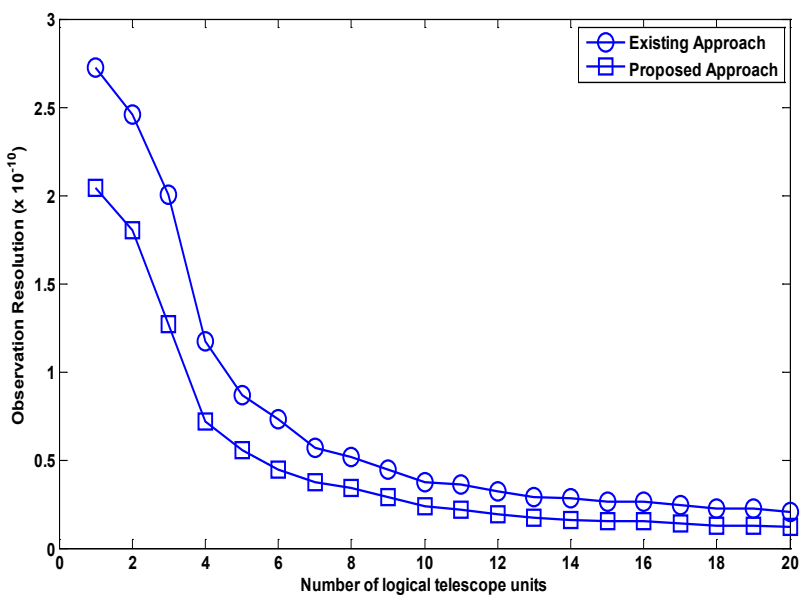

Figure 7: Simulation results obtained for observation resolution

\section{Conclusion}

This paper recognizes that the future conduct of underwater neutrino astronomy has significant research and knowledge value prospects. The knowledge prospect advances scientific research and knowledge. The identified aspects constitute areas where additional research is required. The paper describes the synergy between underwater astronomy and other applications in computing and scientific investigations. In addition, the performance evaluation examines performance improvements that can be obtained by using the proposed synergistic approach. This is done using computing and observation related metrics. The computing related metrics are the PUE and accessible computational resources. The observation related metric is the observation resolution. Investigation shows that the use of the collaborative approach improves accessible computational resources, PUE and resolution by $48.8 \%, 1.6 \%$ and $41.3 \%$ on average, respectively.

\section{Conflict of Interest}

The authors declare no conflict of interest.

\section{Acknowledgment}

The authors acknowledge the financial support of the University of Johannesburg.

\section{References}

[1] J. Baars, T.Beasley, D. Bisikalo, G.Bladon, M.Burton, A.G.de Castro, L.L. Christensen, G.Giovannini, J.M.van der Hulst, C.Keller, A.M. Magalhães, M.Rosenberg and F. Snik, 'From Medicine to Wi-Fi : Technical Applications of Astronomy to Society', (eds) B.Downer, M.Burton, E.van Dishoeck and P.Russo, International Astronomical Union, 2019, [Online] https://www.iau.org/static/archives/announcements/pdf/ann19022a.pdf, Accessed August 4, 2020.

[2] M. Backes, R. Evans, E.K. Kasai, and R. Steenkamp, 'Status of Astronomy in Namibia', Afr. Rev. Phys. 13, 90-95, 2018, eprint 1811.01440.

[3] M.Rosenberg, P.Russo, G.Bladon and L.L.Christensen, 'Why is Astronomy Important?' [Online], https://arxiv.org/ftp/arxiv/papers/1311/1311.0508.pdf, 2013, Accessed, August 4, 2020.

[4] D.Farrah, K.E. Smith, D.Ardilla, C.M.Bradford, M.Dipirro, C.Ferkinhoff, J.Glenn, P.Goldsmith, D.Leisawitze, T.Nikola, N.Rangwala, S.A.Rinehart, J.Staguhn, M.Zemcov, J.Zmuidizinas, J.Bartlett, S.Carey, W.J.Fisher, J.Kamenetz, J.Kartaltepe, M.Lucy, D.C.Lis, L.Locke, E.L.Rodriguez, M.MacGregor, E.Mills, S.H.Moseley, E.J.Murphy, A.Rhodes, M.Richter, 
D.Rigopoulos, D.Sanders, R.Sankrit, G.Savini, J.D.Smith and S.Sherwalt, 'Review: - Far Infrared Instrumentation and Technological Development for the next decade', Journal of Astronomical Telescopes, Instruments and Systems, $\quad \mathbf{5}(2), \quad$ 020901-020901, 2019 , https://doi.org/10.1117/1.JATIS.5.2.020901

[5] United Nations, 'Exploring Space Technologies for Sustainable development and the benefits of international research collaboration in this Context Report of the Secretary - General', Commission on Science and Technology for Development, Twenty - Third Session, Geneva, 23-27 March 2020, Item 3(b) of the Provisional Agenda, https:/unctad.org/system/files/officialdocument/ecn162020d3amend1_en.p df

[6] C.Walker, D.Chingo and S.Dubow, 'Karoo Futures: Astronomy in Place and Space - Introduction', Journal of Southern African Studies, 45(4),627 - 639, 2019, https://doi.org/10.1080/03057070.2019.1654664

[7] M.Boehmer, J.Bosna, D.Brussow, L.Farmer, C.Fruck, R.Gernhauserm, A.Gartner, D.Grant, F.Henningsen, S.Hiller, M.Hoch, K.Holzapfal, R.Jenkyns, Ni, Khera, K.Krings, C.Kopper, I.Kulin, K.Leismuller, J.Little, P.Macroun, J.Michel, M.Morley, L.Papp, B.Pirenne, C.Qiu, I.C.Ree, E.Resconi, A.Round, A.Ruskey, C.Spannfellner and M. Traxler, 'STRAW (STRings for Absorption Length in Water) : Pathfinder for a neutrino telescope in the deep Pacific Ocean', Journal of Instrumentation, 14, 1-30, P02013, 2019, https://doi.org/10.1088/1748-0221/14/02/P02013

[8] S.Aiello, S.E.Akrame, F.Ameli, E.G.Anassontzis, M.Andre, G.Androulakis, M. Anghinolfi, G.Anton, M.Ardid, J.Aublin, T.Avigtals, C.Bagatelas, G.Barbarino, B.Baret, J.Barrios-Marti, A.Belias, E.Berbee, A.van der Berg and J.Zuniga, 'Sensitivity of the KM3Net/ARCA neutrino telescope to point - like neutrino sources', Astro-particle Physics, 111, 100 - 110 ,2019, https://doi.org/10.1016/j.astropartphys.2019.04.002

[9] C.Spiering, 'History of high energy neutrino astronomy', International Conference on History of the Neutrino: 1930-2018 Paris, France, September 5-7, 2018, (2019), ISBN: 9791096879090

[10] V.McBride, R.Venugopal, M.Hoosain, T.Chingozha and K.Govender, 'The Potential of Astronomy for Socioeconomic Development in Africa', Nature Astronomy, 2, 511-514, 2018, https://doi.org/10.1038/s41550-018-0524-y

[11] M.Pović, M.Backes, P. Baki, D.Baratoux, S.B Tessema, Z.Benkhaldoun, M.Bode, N.A. B. Klutse, P.Charles, K. Govender, E.van Groningen, E. Jurua, A.Mamo, S.Manxoyi, V.McBride, J.Mimouni, T.Nemaungani, P. Nkundabakura, B.Okere, S.Saad, P.C. Simpemba, T.Walwa and A.Yilma,' Development in astronomy and space science in Africa', Nature Astronomy, 2, 507- 510, 2018, https://doi.org/10.1038/s41550-018-0525-x

[12] AA Periola and O.E. Falowo, 'Instrumentation Location Diversity Paradigm for Future Astronomy Observations, Wireless Personal Communications, 103, 2475-2499, 2018, https://doi.org/10.1007/s11277-018-5940-x

[13] African Union, 'African Space Strategy - For Social, Political and Economic Integration', [Online] https://au.int/sites/default/files/documents/37434-doc au space_strategy_isbn-electronic.pdf, Accessed 31/07/2020.

[14] J.Retre, P.Russo, H.Lee, E.Penteado, S.Salimpour, M.Fitzgerald, J.Ramchandani, M.Possel, C.Scorza, L.Lindberg, E.Arends, S.Pompea and W.Schrier, 'Big Ideas in Astronomy - A Proposed Definition of Astronomy Literacy', https://www.iau.org/static/archives/announcements/pdf/ann19029a.pdf, May 2019, Accessed August 31, 2020.

[15] M.Gastrow and T.Oppelt, 'Big Science and human development-what is the connection?' South African Journal of Science, 114(11/12), , 1-7, November/December 2018, DOI: https://doi.org/10.17159/sajs.2018/5182

[16] M.J.Sousa, 'Skills to Boost Innovation - In the Context of Public Policies', SWS Journal of Social Sciences and Art, 1, 90 - 103 , 2019, https://doi.org/10.35603/ssa2019/issue 1.09

[17] S.Klein, 'Invest in neutrino astronomy', Nature, 533, 462 - 464, 2016, doi: $10.1038 / 533462 \mathrm{a}$

[18] C.Fruck and I.C. Rea, 'STRAW: STRings for Absorption length in Water', 36TH International Cosmic Ray Conference, ICRC 2019, 358, 1-9, PoS, (ICRC2019) 890,July 24TH - August 1ST, 2019, Madison, WI, U.S.A, http://inspirehep.net/record/1701337

[19] A.D.Thaler and D.Shiffman, 'Fish tales: Combating Fake Science in Popular Media', Ocean \& Coastal Management, 115, 88-91, 2015, https://doi.org/10.1016/j.ocecoaman.2015.04.005

[20] V.Scribner, 'Such Monsters Do Exist in Nature? Mermaids, Tritons and the Science of Wonder in Eighteenth Century Europe', Itinerario, 41(3), 507 538, 2017, Cambridge University Press, DOI: https://doi.org/10.1017/S0165115317000663

[21] G.Fauville, 'Ocean Literacy in the Twenty - First Century' in Exemplary Practices in Marine Science Education: A Resource for Practitioners and Researchers (eds), G.Fauville, D.L.Payne, M.E.Marrero, A. Andersson and
F.Crouch, Springer, 2019, 3-11, DOI:10.1007/978-3-319-90778-9

[22] J.E.Welsh, P.Steenhuis, K.R.Moraes, J.V.D.Meer, D.W.Thieltges, and C.P.D.Brussard, 'Marine Virus Predation by non - host organisms', Scientific Reports, 2020, 10(1),1 -9, 5221, DOI: 10.1038/s41598-02061691-y

.[23] D.L.Kirchman, 'A marine virus as foe and friend', Nature Microbiology, 5, 982 - 983, 2020, DOI https://doi.org/10.1038/s41564-020-0764-3

[24] E.Laanto, 'Viruses - The Invisible Majority of the Oceans', 05 Feb 2019, [Online] Accessed 26/07/2020, https://microbiologysociety.org/publications/pastissues/oceans/article/viruses-the-invisible-majority-of-the-oceans.html

[25] A.D.Avrorin, A.V.Avrorion, R.Bannash, I.A. Belolaptikov, V.R.Brudanin, N.M.Budner, N.S.Gorshkov, A.A. Doroshenko, G.V.Domogatsky, R.Dvornicky, A.N.Dyachok, Zh.A.M.Dzhilikibaev, L.Fajt, S.V.Fialkovsky, A.R.Gafarov, K.V.Golobkov, T.I. Gres, K.G.Kebkal, O.G.Kebkal, E.V.Khramov, M.M.Kolbin, K.V.Konischea, A.V.Korobchenko, A.P.Kosheckin, V.A.Kohzim, M.V.Krughov, M.K.Kryukov, V.F.Kulepov, D.A.Kuleshov, M.B.Milenin, R.A.Mirgazov, V.Nazari, A.I.Panfilov, D.P.Petukhov, E.N.Phikovsky, M.I.Rozamov, E.V.Rjabov, V.D.Rushay, G.B.Safronov, F.Simkovic, A.V.Skurichin, B.A.Shaybonov, A.G.Solovjev, M.N.Sorokovikov, A.V.Skurichin, B.A.Shaybonov, A.G.Solovjev, M.N. Sorokovikov, M.D.Shelepov, G.V.Suvrova, I.Shtekl, V.A. Tabolenko, B.A.Tarashanky, S.A.Yakolev, and A.V.Zagorodnikov, 'Spatial Positioning of Underwater Components for Baikal - GVD', EPPJ Web of Conferences, 207, $1-4, \quad 07004, \quad 2019 ; \quad$ VLVnT-2018, https://doi.org/10.1051/epjconf/201920707004.

[26] M. Sanguineti, 'ANTARES and KM3Net: The Latest Results of the Neutrino Telescopes in the Meditterranean', Universe, 2019, 5(65), $1-9$, doi:10.3390/universe5020065

[27] B.C.O’Leary, and C.M.Roberts, 'Ecological Connectivity across ocean depths: Implications for Protected Area Design', Global Ecology and Conservation, 15, 1-10, 2018, e00431, https://doi.org/10.1016/j.gecco.2018.e00431

[28] R.Garcia and N.Gracias, T.Nicosevici, R.Prados, N.Hurtos, R.Campos, J.Escartin, A.Elibol, R.Hegedus and L.Neumann, 'Exploring the Seafloor with underwater robots', Chapter 4, in Land, Sea \& Air (eds) A.M.Lopez, A.Imiya, T.Pajdla, and A.M. Alvarez, Feb 10, 2017, https://doi.org/10.1002/9781118868065.ch4

[29] V.Vogler, S.Schneider and J. Willmann, 'High-Resolution Underwater 3-D Monitoring Methods to Reconstruct Artificial Coral Reefs in the Bali Sea: A Case Study of an Artificial Reef Prototype in Gili TrawanganIn Journal of Digital Landscape Architecture , 4, 275-289. doi:10.14627/537663030.

[30] F.Ferreira, and D.Machado, G.Ferri, S.Dugelay and J.Potter, 'Underwater Optical and Acoustic Imaging: A Time for Fusion? A Brief overview of the State of the Art, Science and Technology' Organization Centre for Maritime Research and Experimentation, Reprint Series, June 2019, CMRE -PR2019 - 078, doi: 10.1109/OCEANS.2016.7761354

[31] Underwater Acoustic Cameras, https://dosits.org/galleries/technologygallery/observing-and-monitoring-marine-animals/underwater-acousticcameras/

[32] Y.Wang, Y.Ji, H.Woo, Y.Tamara, H.Tsuchiya, A.Yamashita and H.Asama, 'Rotation Estimation of Acoustic Camera Based on illuminated Area in Acoustic Image', IFAC PapersOnline, 52(21), 163 - 168, 2019, https://doi.org/10.1016/j.ifacol.2019.12.301

[33] M.Thomas, B.Martin, K.Kowarski, B.Gaudet and S.Matwin, 'Marine Mammal Species Classification using Convolutional Neural Networks and a Novel Acoustic Representation', in U.Brefeld, E.Fromont, A.Hotho, A.Knobbe, M.Maathuis, and C.Robardet (eds), Machine Learning and Knowledge Discovery in Databases, ECML, PKDD 2019, Lecture Notes in Computer Science, 11908, 290-305, 2019, DOIhttps://doi.org/10.1007/9783-030-46133-1_18

[34] 3D Acoustic Camera 3D Array [Online] https://www.caesystems.de/fileadmin/CAEpage/Datenblaetter/datasheet-acoustic-camera3D-array.pdf, Accessed: Jan 09, 2020.

[35] R. Bailey, 'The Best Underwater Cinema Cameras: An overview of cinema cameras for underwater use',[Online] Available: https://www.uwphotographyguide.com/cinema-cameras-underwater-review, Accessed: Jan 09, 2020.

[36] C.Pellegrino, 'The Trigger and Data Acquisition System (TriDAS) of the KM3Net experiment', Nuovo Cim.C, 39(1), 1-4, 2016, 10.1393/ncc/i2016$16250-9$

[37] M.Favaro, T.Chiarusi, F.Giacomini, M.Manzali, A.Margiotta and C.Pellegrino', The Trigger and Data Acquisition System for the KM3NetItalia Towers', EPJ Web of Conferences, 116(05009), 05009-15, 2016, DOI: $10.1051 /$ epjconf/201611605009 
[38] A.M.Smith, R.Pike, W.O'Mullane, F.Economov, A.Bolton, I.Momcheva, A.E.Bauer, B.Becker, E.Bellm, A.Conmolly, S.M.Crawford, N.Hathi, P. Melchior, J.Peek, A.Solmaz, R.Thomson, E.Tollerud, and D.W.Liska, 'Astronomy should be in the clouds', In Bulletin of the American Astronomical Society, 51, 55, 2019, arXiv: 1907.06320

[39] A.A Periola, 'Incorporating diversity in cloud-computing: a novel paradigm and architecture for enhancing the performance of future cloud radio access networks', Wireless Networks, 25(7), 3783-3803, 2019, , DOIhttps://doi.org/10.1007/s11276-018-01915-2

[40] A.A Periola, A.A Alonge and KA Ogudo, 'Architecture and System Design for Marine Cloud Computing Assets', The Computer Journal, 63(6), 927 941, https://doi.org/10.1093/comjnl/bxz169

[41] J.Roach, 'Microsoft's Undersea Data Centre Helps the hunt for a COVID 19 Vaccine', [Online], https://news.microsoft.com/innovationstories/project-natick-covid-19/, June 16, 2020, Accessed: 27/07/2020.

[42] N.Klein, 'Maritime Autonomous Vehicles within the International Law Framework to Enhance Security International Law Studies', Maritime Autonomous Vehicles, 95, 244-271, 2019, https://digital-

commons.usnwc.edu/cgi/viewcontent.cgi?article $=2907 \&$ context=ils, ISSN: 2375-2831

[43] T.Prodan, J.Kasum, M.Stosic, and C.Ugrin, 'Security Challenges and Guideline Proposals for the Development of Underwater Security', National Security and the Future, 1-2(20), 2019, 71 - 84, https://hrcak.srce.hr/231824

[44] M. Hellyer, 'the Cost of Defence: ASPI Defence Budget Brief', 2019 - 20, Australian Strategic Policy Institute, 2019, http://nla.gov.au/nla.obj770022340

[45] N.J.Bernett, A.M.Montemayor, J.Blythe, J.J.Silver, G.Singh, N.Andrews, A.Calo, P.Christie, A.D.Franco, E.M.Finkbeiner, S.Gelgich, P.Guidetti, S.Harper, N.Hotte, J.N.Kittinger, P.C.Bilton, L.Lister, R.L.Lama, E.McKinley, J.Scholtens, A.M.Solas, M.Sowman, N.T.Alvarez, L.C.L.Teh, M.Voyer and U.R.Sumaila, 'Towards a sustainable and equitable blue economy', Nature Sustainability, 2, 991-993, 2019, DOI: https://doi.org/10.1038/s41893-019-0404-1.

[46] I.Ertor, and M. Hajimichael, 'Editorial: Blue degrowth and the politics of the sea: rethinking the blue economy', Sustainability Science, 15, 1-10, 2020, DOI: https://doi.org/10.1007/s11625-019-00772-y.

[47] I. Lukic and M.Rabaut, 'Marine Spatial Planning to Create Space for a sustainable economy', [Online] https://www.gstic.org/inspiration/marinespatial-planning-sustainable-blue-economy/ Jan 27, 2020, Accessed July 28, 2020.

[48] Kira Gee, 'The Ocean Perspective' in Marine Spatial Planning - Past, Present and Future (eds) Jacek Zaucha, and Kira Gee, 2018, 23 - 45, DOI 10.1007/978-3-319-98696-8

[49] K.G.Balasi, D.Lenis, M.Maniatis, N.Moragos, and G.Stavropoulos, 'A Method for measuring the optical parameters of Deep Sea Water', frontiers in Physics, 6, Nov 2018, Article 132, 111, https://doi.org/10.3389/fphy.2018.00132

[50] C.Spiering, 'History of Neutrino Astronomy and Neutrino Telescopes', 2018, [Online], neutrinohistory2018.in2p3.fr/talks/07_spiering.pdf, Accessed $28 / 07 / 2020$.

[51] R.Retzlaff and C.LeBleu, 'Marine Spatial Planning: Exploring the Role of Planning Practice and Research', Journal of Planning Literature, 33(4) 466491, 2018, https://doi.org/10.1177/0885412218783462

[52] C.F.Santos, C.N.Ehler, T.Agardy, F.Andrade, M.K.Orbach, and L.B.Crowder, 'Marine Spatial Planning 'in World Seas: an environmental evaluation, Academic Press, 571-592, 2019, eBook ISBN: 9780128052037

[53] Rosanna Carver, 'Lessons for blue de-growth from Namibia's emerging blue economy', Sustainability Science, 15, 131-143, 2020, DOI: https://doi.org/10.1007/s11625-019-00754-0.

[54] I.O.Yarwood, N.I.Kadagi, N.A.F.Miranda, J.Uku, I.O.Elegbede and I.J.Adewumi, 'The Blue Economy - Cultural Livelihood - Ecosystem Conservation Triangle: The African Experience', Frontiers in Marine Science, 7(586), 1-18, 2020, https://doi.org/10.3389/fmars.2020.00586

[55] C.M.Rogerson and J.M.Rogerson, 'Emergent Planning for South Africa's blue economy: Evidence from Coastal and Marine Tourism', Urbani izziv, 30, 24-36, Supplement, 2019, DOI: 10.5379/urbani-izziv-en-2019-30supplement-002

[56] R.M. Roettenbacher, 'Interferometry with Meter - Class Telescopes', 2019, Contrib. Astron. Obs. Skalnat'e Pleso, 49, 97-106, https://ui.adsabs.harvard.edu/abs/2019CoSka..49...97R/abstract

[57] G.Bourdarot, H. G.deChatellus and J.P. Berger, 'Toward a large bandwidth photonic correlator for infrared heterodyne interferometer: A first laboratory proof of concept', Astronomy and Astrophysics, 639, A53, 1-7, https://doi.org/10.1051/0004-6361/201937368

www.astesi.com
[58] Y.Yamato, 'Server Selection, Configuraton and Reconfiguration Technology for Iaas Cloud with Multiple Server Types', Journal of Network and Systems Management, 26, 339-360, 2018, DOI: https://doi.org/10.1007/s10922-0179418-Z

[59] J.Kyathsandra, and C. Rego, 'The Efficient Data Center: Improving Datacenter Efficiency Through Intel Technologies and High Ambient Temperature Operation', [Online] https://www.intel.co.jp/content/dam/doc/technology-brief/efficientdatacenter-high-ambient-temperature-operation-brief.pdf, Accessed September 16, 2020.

[60] Z.Zhou, 'GreenEdge : Greening Edge Datacenters with Energy-Harvesting IoT devices', IEEE International Conference on Network Protocols, $8-10$ Oct 2019, 1-6, Chicago IL, USA, USA, DOI: 10.1109/ICNP.2019.8888103

[61] U.F Katz, 'Cherenkov light imaging in astroparticle physics', Nuclear Instruments and Methods in Physics Research Section A: Accelerators, Spectrometers, Detectors and Associated Equipment, 952, 161654, 2020, DOI: 10.1016/j.nima.2018.11.113 